\title{
Alteraciones neuropsicológicas en alcohólicos: un estudio exploratorio
}

\author{
Natalia Landa ${ }^{1}$, Javier Fernández-Montalvo ${ }^{2}$, Javier Tirapu-Ustarroz ${ }^{3}$, José J. López-Goñiª , Ainhoa Castillo², \\ IÑAKI LOREA ${ }^{4}$ \\ 1) Centro de Salud Mental de Tudela (Navarra) \\ 2) Departamento de Psicología y Pedagogía. Universidad Pública de Navarra \\ 3) Servicio de Neuropsicología. Clínica Ubarmin. Pamplona \\ 4) Fundación Proyecto Hombre de Navarra \\ Enviar correspondencia a: \\ Natalia Landa. Centro de Salud Mental de Tudela. C/Gayarre s/n. Tudela. Navarra. E-mail: nataliland@retena.net
}

\section{RESUMEN}

En este estudio se evaluó la presencia de alteraciones neuropsicológicas en 50 pacientes alcohólicos, en comparación con un grupo de control de 50 sujetos de la población normal, apareados en edad, sexo y nivel socioeconómico. Para ello, se aplicó una batería neuropsicológica compuesta por la Escala de Memoria de Wechsler (subtest: control mental, dígitos, localización espacial y memoria lógica), el Trail Making Test de la batería Halstead-Reitan, el test de dibujo de una Figura Compleja de Rey, el test de palabras y colores de Stroop y el subtest de evocación categorial del Test Barcelona. Los resultados obtenidos reflejaron que la ejecución en la mayoría de las pruebas era equiparable en ambos grupos. Las únicas diferencias significativas entre los grupos se observaron en el subtest de memoria lógica, tanto en el recuerdo inmediato como diferido, y en el tiempo empleado en la ejecución de la copia de la Figura de Rey. En ambos casos la muestra de alcohólicos presentaba una peor ejecución, con una afectación de la memoria en cuanto a la capacidad de realizar nuevos aprendizajes. Los resultados descartaron la existencia de un déficit cognoscitivo generalizado en los pacientes alcohólicos. La ausencia de otras alteraciones cognoscitivas, sobre todo en las funciones ejecutivas, contrasta con los hallazgos de otras investigaciones.

Palabras-clave: Alcoholismo. Déficit neuropsicológico. Evaluación.

\section{INTRODUCCIÓN}

E estudio del déficit cognoscitivo asociado al consumo de alcohol cuenta con una larga tradición. Ya a finales del siglo XIX se describió el síndrome de Wernicke-Korsakoff asociado al consumo crónico de alcohol, que cursa, básicamente, con un deterioro grave de la memoria. Sin embargo, ha sido en las últimas décadas cuando se han desarrollado los

\section{ABSTRACT}

This study evaluates the presence of neuropsychological disorders in 50 alcoholic patients compared with a control group of 50 subjects from the normal population, matched on age, sex, and socioeconomic level. The neuropsychological assessment battery comprised the Wechsler Memory Scale (subtest: mental control, digits, space localisation and logical memory), the Trail Making Test from the Halstead-Reitan Battery, the Rey Complex Figure drawing test, the Stroop words and colours test and the Barcelona Test subtest on categorical evocation. The results showed that carrying out the tasks was similar for both groups. The only significant differences between the groups were observed in the logic memory subtest, both in the immediate and in the delayed memory, and in the time taken to copy the Rey figure. In both cases, the alcoholic patients fared worse, with memory being affected in respect of the ability to learn new skills. The results do not show the existence of a generalised cognitive deficit in alcoholic patients. The absence of other cognitive distortions, mainly in the executive functions, contrast with the findings of previous studies.

Keywords: Alcoholism. Neuropsychological deficit. Assessment. principales estudios sobre los déficit neuropsicológicos en el alcoholismo, asociados, en gran medida, a los nuevos modelos explicativos del funcionamiento cerebral, así como a la aparición de técnicas más precisas para su estudio (Tirapu, Landa y Lorea, 2004).

En una reciente revisión sobre el tema (Landa, Fernández-Montalvo y Tirapu, 2004) se concluye que la mayoría de las investigaciones señalan la existencia de un deterioro en la memoria de trabajo y en las fun- 
ciones ejecutivas. Más en concreto, el mayor acuerdo en los diferentes estudios se observa en la alteración de la memoria a corto plazo (Ambrose, Borden y Whelan, 2001; Brandt, Butters, Ryan y Bayog, 1983; Mann, Günter, Setter y Ackerman, 1999; Nixon y Bowly, 1996; Query y Berger, 1980; Tarquini y Masullo, 1981).

Sin embargo, existe menos coincidencia en cuanto a los hallazgos encontrados en el resto de las alteraciones mnésicas. En algunos estudios, se encuentra que la capacidad de memoria general está afectada, mientras que la memoria verbal se mantiene sin alteraciones (Acker, Ron, Lishman y Shaw, 1984; Hightower y Anderson, 1986; Nixon, Kujawski, Parsons y Yohman, 1987). En otros, se observa una mayor conservación de la memoria visual (Brandt et al., 1983; Mann et al., 1999; Riege, Tomaszewewski, Lanto y Metter, 1984), aunque con resultados contradictorios entre los mismos (Acker et al., 1984; Hightower y Anderson, 1986; Leber, Jenkins y Parsons, 1981; Yohman y Parsons, 1985). Por último, los estudios que utilizan una terminología más precisa coinciden en señalar el déficit en la memoria de trabajo, mientras que la memoria declarativa se hallaría preservada (Ambrose et al., 2001; Sullivan, Rosenbloom y Pfefferbaum, 2000; Sullivan, Fama, Rosenbloom y Pfefferbaum, 2002).

Por lo que se refiere a las funciones ejecutivas, los estudios llevados a cabo hasta la fecha muestran un mayor grado de acuerdo. De hecho, se observa una afectación en este tipo de funciones, implicadas en la resolución de situaciones novedosas y que incluyen aspectos tan diversos como la planificación, la abstracción, la inhibición de respuestas irrelevantes, la flexibilidad mental, la generación de conceptos, los ensayos mentales y el reconocimiento del logro (Corral, Rodríguez y Cadaveira, 2002; Giancola y Moss, 1998; Ihara, Berrios y London, 2000; Moshely, Georgiou y Kahn, 2001; Nöel, Van der Linden, Schmidt, Sferraza, Hanak et al., 2001; Ratti, Bo, Giardini y Soragna, 2002; Sullivan, Mathalon, Zipursky, Kersteen-Tucker, Knight, y Pfefferbaum, 1993).

Asimismo, existen estudios que constatan la alteración de la capacidad de atención alternante (Beatty, Katzung, Moreland y Nixon, 1995; Parsons y Leber, 1981; Sullivan et al., 2002) y de las habilidades visoconstructivas (Beatty et al., 1995; Dawson y Grant, 2000; Sullivan et al., 2000, 2002; Tivis, Beatty, Nixon y Parsons, 1995).

En resumen, aunque con excepciones, los resultados encontrados hasta la fecha constatan la existencia de diferentes alteraciones neuropsicológicas en los alcohólicos. A partir de esta evidencia, los investigadores han tratado de explicar cual es el substrato cerebral que subyace a estos hallazgos, y se han planteado tres hipótesis fundamentales sobre el patrón de deterioro cognoscitivo presente en el alcoholismo: (1) la hipótesis de la vulnerabilidad del hemisferio dere- cho; (2) la hipótesis del daño cerebral difuso; y (3) la hipótesis de la vulnerabilidad diferencial del lóbulo frontal.

Según la hipótesis de la vulnerabilidad del hemisferio derecho, el consumo de alcohol provoca cambios neurodegenerativos o electrofisiológicos que son más pronunciados en el hemisferio derecho (Bertera y Parsons, 1978; Jenkins y Parsons, 1981; Miglioli, Butchtel, Campanini y Derisio, 1979; Tsagareli, 1995). Para los defensores de la hipótesis del daño cerebral difuso (Beatty, Hames, Blanco, Nixon y Tivis, 1996; Parsons, 1987; Tivis et al., 1995), el abuso crónico de alcohol produce efectos neurotóxicos en todo el cerebro, lo que da lugar a una afectación cognitiva leve o moderada. Por último, según la hipótesis de la vulnerabilidad diferencial del lóbulo frontal (o del circuito fronto-límbico-diencefálico), esta región cerebral, por sus especiales características, es más sensible a los efectos tóxicos del alcohol (Ciesielki, Waldorf y Jung, 1995; Nöel et al., 2001; Ratti, Soragna, Sibilla, Giardini, Albergati et al., 1999; Tarter, 1975, 1976).

En cualquier caso, desde una perspectiva clínica, el estudio neuropsicológico de los pacientes alcohólicos cobra una especial relevancia. El perfil cognoscitivo de estos pacientes puede constituir un factor pronóstico importante y señalar las líneas terapéuticas más adecuadas (McCrady y Smith, 1986). En este artículo se presentan los resultados de la evaluación neuropsicológica de una muestra de alcohólicos que acuden a consulta en busca de tratamiento, y se comparan los resultados con los obtenidos por un grupo de control de la población normal. Asimismo, se valora la posible influencia de la gravedad de la adicción en el déficit hallado.

\section{MÉTODO}

\section{Muestra}

La muestra de este estudio está compuesta por 100 sujetos (50 alcohólicos y 50 personas de la población general). Todos ellos mostraron su consentimiento para participar en el estudio, tras haber sido ampliamente informados. Las principales características sociodemográficas de ambos grupos aparecen descritas en la tabla 1.

Por lo que se refiere a la muestra clínica, se trata de pacientes que acudieron en busca de ayuda terapéutica al programa de tratamiento ambulatorio del alcoholismo de Proyecto Hombre de Navarra, durante el período comprendido entre noviembre de 2003 y agosto de 2004. De acuerdo con los criterios de admisión al estudio, los pacientes tenían que: a) cumplir los criterios diagnósticos del DSM-IV-TR (American 


\section{TABLA 1. CARACTERÍSTICAS SOCIODEMOGRÁFICAS DE LA MUESTRA}

\begin{tabular}{|c|c|c|c|}
\hline Variables & $\begin{array}{c}\text { Grupo de alcohólicos } \\
\mathrm{N}=50 \text { - Media (DT) }\end{array}$ & $\begin{array}{l}\text { Grupo de control } \\
\mathrm{N}=50 \text { - Media (DT) }\end{array}$ & $t$ \\
\hline Edad Media & $43,89(7,7)$ & $42,62(9,6)$ & 0,73 \\
\hline Variables & $\begin{array}{c}\text { Grupo de alcohólicos } \\
\qquad N=50-N(\%)\end{array}$ & $\begin{array}{l}\text { Grupo de control } \\
\qquad N=50-N(\%)\end{array}$ & $X^{2}$ \\
\hline \multicolumn{4}{|l|}{ Sexo } \\
\hline Hombre & $38(76 \%)$ & $38(76 \%)$ & - \\
\hline Mujer & $12(24 \%)$ & $12(24 \%)$ & \\
\hline \multicolumn{4}{|l|}{ Estado civil } \\
\hline Soltero & $10(20 \%)$ & $13(26 \%)$ & \\
\hline Casado/conviviendo & $25(50 \%)$ & $32(64 \%)$ & $6,25^{*}$ \\
\hline Separado/divorciado & $15(30 \%)$ & $5(10 \%)$ & \\
\hline \multicolumn{4}{|l|}{ Nivel cultural } \\
\hline Sin estudios/estudios primarios & $28(56 \%)$ & $22(44 \%)$ & \\
\hline Estudios secundarios & 17 (34\%) & $20(40 \%)$ & 1,66 \\
\hline Estudios universitarios & $5(10 \%)$ & & $8(16 \%)$ \\
\hline \multicolumn{4}{|l|}{ Situación laboral } \\
\hline En activo & $19(38 \%)$ & $43(86 \%)$ & \\
\hline Desempleado & $13(26 \%)$ & $4(8 \%)$ & $24,8 * *$ \\
\hline ILT/Pensionista & $18(36 \%)$ & $3(6 \%)$ & \\
\hline${ }^{*} p<0,05 ;{ }^{* *} p<0,001$ & & & \\
\hline
\end{tabular}

Psychiatric Association, 2000) para la dependencia alcohólica; b) tener una puntuación igual o superior a 11 en la versión española (Rodríguez-Martos y Suárez, 1984) del Müncher Alkoholismus Test (MALT) (Feuerlein, Ringer, y Kufner, 1977); c) tener una edad comprendida entre los 18 y 65 años; d) mantener la abstinencia del alcohol durante las tres semanas previas a la evaluación; y e) participar voluntariamente en el estudio después de haber sido debidamente informado.

Como se puede observar en la tabla 1, la edad media de los alcohólicos es de 43 años, con un rango que oscila entre 29 y 57 años. La muestra está compuesta por 38 varones (el 76\%), y 12 mujeres (el $24 \%)$. Las únicas diferencias significativas entre los alcohólicos y el grupo de control se encontraron en el estado civil -con un mayor porcentaje de solteros o separados en la muestra de alcohólicos- y en la situación laboral -con un mayor número de casos en situación de desempleo o de incapacidad laboral transitoria en el caso de los alcohólicos-.

Las principales características de consumo de los alcohólicos seleccionados eran las siguientes: la puntuación media en el MALT era de 29,5 puntos $(D T=8,2)$, con un rango comprendido entre 11 y 50 puntos. El consumo de alcohol se caracterizaba, en términos medios, por ser frecuente (6-7 días/semana), con una media de 216,7 gramos diarios ( $D T=123,6$; rango: 50-640 gramos diarios). La antigüedad de la dependencia alcohólica se remontaba, como media, a casi 12 años $(D T=6,8)$, antes de acudir en busca de tratamiento. En ninguna de las variables de consumo de alcohol se observaron diferencias estadísticamente significativas entre los hombres y las mujeres de la muestra alcohólica (tabla 2). Esta ausencia de diferencias en función del sexo, que contrasta con la bibliografía previa, está relacionada, probablemente, con el bajo número de mujeres alcohólicas de la muestra.

Por otra parte, el grupo de control estaba compuesto por 50 sujetos seleccionados entre la población normal. Se trataba de personas sanas sin patología mental, que acudían en busca de tratamiento por diferentes problemas traumatológicos leves a la Clínica Ubarmin de Pamplona, y que estaban apareadas en edad, sexo y nivel cultural con las del grupo anterior. Para la selección de este grupo se tuvo un especial cuidado en excluir los sujetos afectados por un traumatismo importante (por ejemplo, un traumatismo cráneo-encefálico). Asimismo, con el objetivo de controlar la presencia de un posible abuso de alcohol, se excluyeron los sujetos que obtenían dos o más puntos en el cuestionario CAGE (Chan, 1994) para el screening del alcoholismo. 
TABLA 2. CARACTERÍSTICAS DEL CONSUMO DE ALCOHOL

\begin{tabular}{|c|c|c|c|c|}
\hline VARIABLES & $\begin{array}{c}\text { TOTAL } \\
\mathrm{N}=50-\text { Media (DT) }\end{array}$ & $\begin{array}{c}\text { HOMBRES } \\
\mathrm{N}=38-\text { Media (DT) }\end{array}$ & $\begin{array}{c}\text { MUJERES } \\
\mathrm{N}=12-\text { Media (DT) }\end{array}$ & $t$ \\
\hline \multicolumn{5}{|l|}{ MALT } \\
\hline MALT-Subjetivo & $18,7 \quad(3,8)$ & $18,8 \quad(3,9)$ & $18,5 \quad(3,9)$ & 0,18 \\
\hline MALT-Objetivo & $10,5 \quad(5,5)$ & $11,1 \quad(5,6)$ & $9,1 \quad(4,8)$ & 1,12 \\
\hline MALT-Total & $29,5 \quad(8,2)$ & $30,1 \quad(8,4)$ & $27,5 \quad(7,6)$ & 0,92 \\
\hline Años de adicción & $11,8 \quad(6,8)$ & $12,7 \quad(7,2)$ & $8,9 \quad(4,5)$ & 1,70 \\
\hline Gramos diarios & $216,7(123,6)$ & $230,9(129,8)$ & $171,6(91,9)$ & 1,46 \\
\hline VARIABLES & $\begin{array}{c}\text { TOTAL } \\
\mathrm{N}=50-\mathrm{N}(\%)\end{array}$ & $\begin{array}{l}\text { HOMBRES } \\
\mathrm{N}=38-\mathrm{N}(\%)\end{array}$ & $\begin{array}{c}\text { MUJERES } \\
\mathrm{N}=12-\mathrm{N}(\%)\end{array}$ & $X^{2}$ \\
\hline \multicolumn{5}{|l|}{ Patrón de consumo } \\
\hline Continuo & $39(78 \%)$ & $31(81,6 \%)$ & $8(66,7 \%)$ & 1,18 \\
\hline Episódico & $11 \quad(22 \%)$ & $7(18,4 \%)$ & $4(33,3 \%)$ & \\
\hline \multicolumn{5}{|c|}{ Frecuencia de consumo } \\
\hline Diario & $36 \quad(72 \%)$ & $29(76,3 \%)$ & $7 \quad(58,3 \%)$ & \\
\hline 4-5 días/semana & $5(10 \%)$ & $3(7,9 \%)$ & $2(16,7 \%)$ & 5,56 \\
\hline 2-3 días/semana & $9(18 \%)$ & $6(15,8 \%)$ & $3(25 \%)$ & \\
\hline
\end{tabular}

\section{Instrumentos de evaluación}

Variables de consumo de alcohol

El Müncher Alkoholismus Test (MALT) (Feuerlein et al. 1977) es una prueba diagnóstica que está compuesta por dos partes. La primera (MALT-O), de carácter objetivo, consta de 7 ítems que son cumplimentados por el terapeuta a partir de los datos procedentes de la exploración clínica, del historial del paciente y de los análisis de laboratorio. Cada respuesta positiva recibe una puntuación de 4 puntos. La segunda parte del instrumento (MALT-S), de carácter subjetivo, consta de 27 ítems auto-administrados, cada uno de ellos con un rango de 0 a 1 puntos. Una puntuación total en el instrumento igual o superior a 11, el punto de corte, sugiere la presencia de un diagnóstico de alcoholismo. En esta investigación se ha utilizado la versión española de Rodríguez-Martos y Suárez (1984).

\section{Variables neuropsicológicas}

Para la valoración del déficit neuropsicológico se aplicó una amplia batería compuesta por pruebas seleccionadas de diferentes test. Se trataba de llevar a cabo una valoración completa de las distintas funciones cognoscitivas, con pruebas ampliamente utilizadas. En la tabla 3 se presenta el resumen de las pruebas utilizadas, así como las funciones que evalúan cada una de ellas.

\section{TABLA 3. PROTOCOLO DE EVALUACIÓN NEUROPSICOLÓGICA}

\begin{tabular}{|ll|} 
Funciones cognoscitivas & Pruebas \\
Atención y memoria de trabajo & $\begin{array}{c}\text { Subtests de la Escala de memoria de Wechsler: } \\
\text { • Control mental } \\
\text { • Dígitos (directo e inverso) } \\
\text { • Localización espacial (directo e inverso) } \\
\text { Batería Halstead-Reitan: } \\
\text { - Trail Making Test B }\end{array}$ \\
\hline Memoria & $\begin{array}{c}\text { Subtests de la Escala de memoria de Wechsler: } \\
\text { • Memoria lógica I y II } \\
\text { Test del dibujo de una figura compleja de Rey (reproducción de memoria) }\end{array}$ \\
\hline Funciones ejecutivas & $\begin{array}{l}\text { Test de palabras y colores de Stroop } \\
\text { Evocación categorial (animales y palabras) }\end{array}$ \\
\hline Habilidades visoconstructivas & Test del dibujo de una figura compleja de Rey (copia) \\
\hline
\end{tabular}


La Escala de Memoria de Wechsler (Wechsler, 1987) es un instrumento útil para la evaluación de los déficit mnésicos en todo tipo de pacientes. En este estudio se incluyeron algunos de los subtest de esta escala: control mental, dígitos, localización espacial y memoria lógica inmediata y demorada. El subtest de control mental evalúa la capacidad para realizar automáticamente una serie de procesos como contar hacia atrás o realizar series de sumas. El subtest de dígitos evalúa la capacidad para repetir una secuencia presentada por vía auditiva. El subtest de localización espacial mide la capacidad para repetir una secuencia presentada visualmente. Estas tres pruebas evalúan la memoria de trabajo, por lo que según las descripciones actuales de este concepto (Baddeley, 1993) constituyen tests de atención. Por lo que se refiere al subtest de Memoria lógica, tanto en su versión de recuerdo inmediato (Memoria lógica I) como de recuerdo demorado (Memoria lógica II), constituyen el tipo de test empleado tradicionalmente para evaluar la memoria verbal o memoria declarativa. En esta prueba se relatan dos breves historias y se le solicita al paciente que las repita de forma inmediata. Posteriormente se pide un nuevo relato de las historias transcurridos 30 minutos desde la primera lectura.

El Trail Making Test (TMT), parte B, forma parte de la Batería Halstead-Reitan (Jarvis y Barth, 1994). Se trata de un test ampliamente utilizado para evaluar la capacidad de atención alternante. Para llevar a cabo la tarea correctamente es necesario realizar una adecuada exploración visual y mantener la atención de forma alternante para unir dos secuencias. La lentitud en la coordinación visomotriz o las dificultades de atención aumentan el tiempo de ejecución.

El Test de la Figura Compleja de Rey (Rey, 1959) consiste en copiar y después reproducir de memoria un dibujo geométrico complejo. La forma y calidad en que el sujeto copia la figura aporta información sobre su actividad y organización perceptiva, mientras que la reproducción efectuada una vez retirado el modelo informa sobre el grado y fidelidad de su memoria visual.

El Test de Palabras y Colores de Stroop (Stroop, 1935) evalúa la capacidad de un sujeto para inhibir una respuesta automática. De esta prueba se obtienen cuatro medidas y una de ellas, la de interferencia, refleja la capacidad del sujeto para clasificar información de su entorno y reaccionar selectivamente a esa información. Esta puntuación se considera una buena medida de las funciones ejecutivas.

El subtest de Evocación Categorial se ha extraído del Programa integrado de exploración neuropsicológica "Test Barcelona" (Peña-Casanova, 1991), que a su vez realiza una adaptación de los test originales: el Test de palabras de Thurstone (1938) y el Test de nombres de animales de Strub y Black (1995). Se trata de una tarea cognitiva que requiere un adecuado funcionamiento de la memoria de trabajo y de las funciones ejecutivas, como la iniciación y las estrategias de búsqueda.

\section{Procedimiento}

Una vez seleccionados los sujetos de la muestra de acuerdo con los criterios previamente indicados, la evaluación de los alcohólicos se llevó a cabo en el marco de la evaluación pretratamiento. No obstante, la exploración neuropsicológica tuvo lugar una vez transcurrido un período de abstinencia inicial de 3 semanas. Este último criterio tenía como objetivo controlar la interferencia de un estado de intoxicación o de la sintomatología abstinencial en el desarrollo de las pruebas. Más en concreto, la evaluación tuvo una duración de dos sesiones. En la primera se recogieron los datos relacionados con las características sociodemográficas y con el consumo de alcohol. En la segunda sesión se aplicó el protocolo completo de evaluación neuropsicológica.

La evaluación del grupo de control procedente de la población normal se llevó a cabo tras una estratificación previa en edad, sexo y clase social. Para la evaluación de este grupo se utilizó una única sesión en la que se aplicó el protocolo completo de evaluación neuropsicológica.

\section{RESULTADOS}

\section{Perfil neuropsicológico}

En este apartado se presentan los resultados obtenidos en las pruebas de evaluación neuropsicológica, así como la comparación de la muestra de alcohólicos con el grupo de control (tabla 4).

Los resultados obtenidos muestran la ausencia de diferencias estadísticamente significativas entre los dos grupos en la práctica totalidad de variables estudiadas. Las únicas diferencias se observan en el subtest de memoria lógica, tanto en la reproducción inmediata como en la diferida, y en el tiempo empleado para la ejecución de la copia de la Figura de Rey. En concreto, el rendimiento en las pruebas que evalúan la memoria -referida, en este caso, a la capacidad para realizar nuevos aprendizajes verbales- era inferior en el grupo de alcohólicos. Además, los alcohólicos empleaban más tiempo en la ejecución de la copia de la figura de Rey.

Por otra parte, cuando se compara el perfil neuropsicológico entre los hombres y las mujeres de la muestra alcohólica, sólo se encuentran diferencias 


\section{TABLA 4. RESULTADOS DE LA EVALUACIÓN NEUROPSICOLÓGICA (1)}

Test

Control mental

Dígitos directos

Dígitos inversos

Dígitos total

Localización espacial, directo

Localización espacial, inversa

Localización espacial, total

Índice de atención, puntuación directa

Índice de atención

Memoria lógica I

Memoria lógica II
Grupo de alcohólicos $\mathrm{N}=50$ - Media (DT)
$4,04(1,21)$
$6,70(2,21)$
$4,96(1,69)$
$11,68(3,28)$
$7,62(1,57)$
$7,04(1,71)$
$14,64(2,68)$
$56,68(10,93)$
$85,08(17,22)$
$20,98(5,98)$
$15,48(6,82)$

Grupo de control $\mathrm{N}=50$ - Media (DT)

$4,32(1,01)$

1,25

$6,54(1,68)$

$5,14(1,78)$

$11,68(2,90)$

$7,66(1,47)$

$7,14(1,85)$

$14,80(2,65)$

$57,34(9,56)$

$85,50(12,48)$

$24,30(6,24)$

$19,40(6,31)$
0,41

0,52

0

1,31

0,28

0,30

0,32

0,14

$2,71 *$

$2,98 *$

${ }^{*} p<0,01$

\section{TABLA 4. RESULTADOS DE LA EVALUACIÓN NEUROPSICOLÓGICA (y 2)}

Test

Stroop, palabra, puntuación directa

Stroop, palabra, puntuación T

Stroop, color, puntuación directa

Stroop, color, puntuación T

Stroop, palabra-color, puntuación directa

Stroop palabra-color puntuación T

Stroop, interferencia directa

Stroop, interferencia, puntuación T

Figura de Rey, copia, puntuación directa

Figura de Rey, memoria directa

Figura de Rey, tiempo de copia en segundos

Evocación categorial, animales 1 minuto

Evocación categorial, palabras

Tail Making Test B segundos

\section{Grupo de alcohólicos $\mathrm{N}=50$ - Media (DT)}

$105,29(16,28)$

$48,53(9,36)$

$67,27(12,86)$

$41,45(8,64)$

$38,86(9,54)$

$43,63(9,25)$

$-2,22(7,44)$

$47,35(7,29)$

$31,14(3,37)$

$18,90(19,67)$

$179,21(81,49)$

$23,80(7,40)$

$34,48(11,45)$

$138,1 \quad(103,33)$
Grupo de control
$\mathrm{N}=50$ - Media (DT)

$\boldsymbol{t}$

$109,88(17,58)$

1,35

$50,92(8,82)$

$69,64(13,98)$

$43,06(9,36)$

$41,92(9,93)$

$46,82(9,94)$

$-1,11(7,30)$

$48,66(6,72)$

$30,12(4,55)$

$17,92(6,64)$

$124,32(51,34)$

$22,58(6,33)$

$33,60(10,54)$

$121,4(91,18)$

${ }^{*} p<0,001$

significativas en la prueba de control mental, con un mejor rendimiento en el caso de los hombres $(t=2,8$; $p<0,001)$. Se trata de una prueba que evalúa funciones relacionadas con la memoria de trabajo y, más en concreto, con el sistema atencional supervisor, e implica procesos de mantenimiento, manipulación e inhibición de la información. Sin embargo, en el resto de tareas que evalúan funciones similares (dígitos y localización espacial), no se confirman estas diferencias. No obstante, debe tenerse en cuenta que esta ausencia de diferencias importantes en función del sexo puede deberse al bajo número de mujeres alcohólicas de la muestra $(\mathrm{N}=12)$.

Asimismo, cuando se han comparado los resultados en función de la edad de la muestra, tampoco se han encontrado diferencias estadísticamente significativas entre los grupos de mayor edad y los pacientes más jóvenes.

Desde una perspectiva más clínica, resulta interesante, al margen de las puntuaciones medias obtenidas, conocer el número de sujetos que presentan un déficit específico en las distintas pruebas. Para ello se han utilizado los baremos disponibles de cada una de ellas. Los resultados obtenidos en este sentido se exponen en la tabla 5.

Por lo que se refiere al índice de atención de la escala Wechsler de memoria, es una puntuación compuesta que incluye las puntuaciones de control mental, dígitos y localización espacial, y que se corrige en 
TABLA 5. TASA DE EJECUCIONES CORRECTAS Y DEFICIENTES EN EL GRUPO CLÍNICOY EN EL GRUPO DE CONTROL

\begin{tabular}{|c|c|c|c|c|c|}
\hline \multirow[b]{2}{*}{ ESCALA } & \multicolumn{2}{|c|}{ GRUPO CLÍNICO } & \multicolumn{2}{|c|}{ GRUPO CONTROL } & \multirow[b]{2}{*}{$x^{2}$} \\
\hline & $\begin{array}{c}\text { Correcto } \\
\text { N (\%) }\end{array}$ & $\begin{array}{l}\text { Déficit } \\
\text { N (\%) }\end{array}$ & $\begin{array}{c}\text { Correcto } \\
\mathbf{N}(\%)\end{array}$ & $\begin{array}{l}\text { Déficit } \\
\text { N (\%) }\end{array}$ & \\
\hline Índice de atención & $33(66 \%)$ & $17(34 \%)$ & $31(62 \%)$ & $19(38 \%)$ & 0,17 \\
\hline \multicolumn{6}{|l|}{ STROOP } \\
\hline Palabra & $43(12,2 \%)$ & $6(8 \%)$ & 46 (92\%) & $4(8 \%)$ & 0,49 \\
\hline Color & $27(55,1 \%)$ & $22(44,9 \%)$ & 33 (66\%) & 17 (34\%) & 1,23 \\
\hline Palabra-color & $39(61,2 \%)$ & $19(38,8 \%)$ & 37 (74\%) & $13(26 \%)$ & 0,59 \\
\hline Interferencia & $41(83,7 \%)$ & $8(16,3 \%)$ & 47 (94\%) & $3(6 \%)$ & 2,67 \\
\hline \multicolumn{6}{|l|}{ Figura de Rey } \\
\hline Copia & $36(28 \%)$ & $14(72 \%)$ & $35(70 \%)$ & $15(30 \%)$ & 0,05 \\
\hline Memoria & $16(32 \%)$ & $34(68 \%)$ & $15(31,3 \%)$ & $33(68,8 \%)$ & 0,01 \\
\hline \multicolumn{6}{|l|}{ Evocación categorial } \\
\hline Animales & $29(42 \%)$ & $21(58 \%)$ & 28 (56\%) & 22 (44\%) & 0,04 \\
\hline Palabras & $29(42 \%)$ & $21(58 \%)$ & $26(52 \%)$ & $24(48 \%)$ & 0,36 \\
\hline
\end{tabular}

función de la edad del sujeto. La media de este índice es de 100. En este estudio se ha considerado que un índice igual o superior a 80 refleja una adecuada ejecución. Como queda reflejado en la tabla 5, no existen diferencias significativas entre ambos grupos con este punto de corte.

Por lo que se refiere al test de palabras y colores de Stroop, se ha considerado como ejecución correcta la obtención de una puntuación típica igual o superior a 40, una vez realizadas las correcciones en función de la edad. Los resultados muestran que el porcentaje de ejecuciones correctas es superior en el grupo de control, aunque las diferencias con la muestra alcohólica no son significativas.

En el test de la figura de Rey se consideran como ejecuciones correctas aquellas que se sitúan por encima del percentil 40. En este caso, las puntuaciones obtenidas se distribuyen de forma muy similar en ambos grupos.

Asimismo, se analizó el tipo de copia en la Figura de Rey y se establecieron dos grupos en función de si el proceso de copia se consideraba correcto (tipo I y II) o se consideraba incorrecto (resto de tipos). Los resultados obtenidos con este criterio muestran que los alcohólicos ejecutaron adecuadamente la copia con mayor frecuencia que el grupo de control (32 ocasiones frente a 26), aunque sin diferencias estadísticamente significativas.

Por último, el test de evocación categorial o fluencia verbal se compone de dos pruebas similares. En ambas se considera como punto de corte del percentil 40. El rendimiento de ambos grupos resulta también similar.

\section{Gravedad de la adicción y déficit neuropsicológico}

Con el objetivo de valorar en qué medida la mayor gravedad de la adicción alcohólica se relacionaba con una mayor presencia de déficit neuropsicológicos, se llevó a cabo un análisis de correlación entre las variables estudiadas.

Los resultados encontrados mostraron una ausencia de correlaciones estadísticamente significativa entre las distintas variables de consumo de alcohol (por ejemplo, la puntuación en el MALT, la edad de inicio, la cantidad y la frecuencia del consumo, la antigüedad de la dependencia o el consumo añadido de otras sustancias) y la ejecución en las pruebas neuropsicológicas. Por tanto, la gravedad de la dependencia alcohólica no se relacionaba con la presencia de alteraciones neuropsicológicas.

\section{DISCUSIÓN}

En este estudio se ha llevado a cabo una evaluación neuropsicológica de pacientes alcohólicos y se han comparado los resultados obtenidos con un grupo de control procedente de la población normal. Los resultados encontrados muestran la ausencia de un déficit cognoscitivo generalizado en la muestra de alcohólicos. Las diferencias encontradas con respecto al grupo de control sólo tienen significación estadística en las pruebas de memoria verbal, tanto inmediata como demorada, y en el tiempo empleado en la figura de Rey. Por tanto, los alcohólicos de la muestra presentan una alteración en la capacidad para reali- 
zar nuevos aprendizajes y un enlentecimiento en los procesos cognitivos implicados en la copia de una figura compleja. Sin embargo, no existen diferencias en el resto de capacidades cognoscitivas estudiadas: capacidad de atención, memoria de trabajo, memoria visual, funciones ejecutivas y habilidades visoconstructivas.

Esta ausencia de alteraciones cognoscitivas importantes contrasta con una parte de la evidencia científica disponible en la actualidad (Beatty et al., 1995; Calvo, 2003; Ihara et al., 2000; Iruarrizaga, MiguelTobal y Cano, 2001; Mann et al., 1999; Moshely et al., 2001; Parsons y Leber, 1981; Ratti et al., 2002). Sin embargo, es coincidente con los resultados de otros estudios en los que tampoco se encuentra un déficit neuropsicológico importante (Eckardt, Stapleton, Rawlings, Davis y Grodin, 1995; Krabbendam, Visser, Derix, Verhey, Hofman et al., 2000) o, en todo caso, sólo una alteración de algunas capacidades específicas (Acker et al., 1984; Brandt et al., 1983; Hightower y Anderson, 1986; Nixon et al., 1987; Riege et al., 1984).

La mayor discrepancia entre los resultados de este estudio y la bibliografía existente reside en no haber encontrado un déficit en la memoria de trabajo, ya que es el resultado más coincidente entre numerosas investigaciones (Ambrose et al., 2001; Becker y Jaffe, 1984; Brandt et al., 1983; Mann et al., 1999; Nixon y Bowly, 1996; Query y Berger, 1980; Sullivan et al., 2002; Tarquini y Masullo, 1981), aunque no todos los estudios confirman este hallazgo (Eckardt et al., 1995; Nöel et al., 2001; Sullivan et al., 2000; Yohman y Parsons, 1985).

La ausencia de un déficit generalizado se puede explicar por algunas de las características de la muestra. Así, la media de edad es relativamente baja (43 años) y el rango es muy amplio (29-65), cuando numerosos estudios evidencian que este factor tiene una gran importancia en cuanto a la magnitud del déficit (Cermak y Peck, 1982; Donovan Kivlahan y Walter, 1984; Ellenberg, Rosenbaum, Goldman y Whitman, 1980; Fein, Bachman, Fisher y Davenport, 1990; Goldman, Williams y Klisz, 1983; Hesselbrock, Weidenman y Reed, 1985; Ryan y Butters, 1980; Tarter y Alterman, 1984). En este sentido, se hace necesario, de cara a futuras investigaciones, tener en cuenta los distintos intervalos de edad de los pacientes y comparar, con muestras más amplias, el rendimiento en las distintas pruebas neuropsicológicas en función de la edad. Por otra parte, se trata de una muestra muy heterogénea en cuanto a la cantidad y el tiempo de consumo y estas variables podrían tener cierta influencia en las alteraciones cognitivas (Beatty, Tivis, Scott, Nixon y Parsons, 2000; Eckardt et al., 1995; Eckardt, File, Gessa, Grant, Guerra et al., 1998; Horner, Waid, Johnson, Latham y Anton, 1999).
Por otra parte, en el caso de la evaluación de las funciones ejecutivas, las pruebas aplicadas presentan una limitación, ya que no permiten descartar totalmente la afectación de estas complejas funciones cognoscitivas. Tanto el subtest de evocación categorial, como el test de Stroop, evalúan habilidades muy específicas dentro de las capacidades del lóbulo frontal. El primero trata de valorar la capacidad de generación de respuestas siguiendo un criterio prefijado y el segundo la capacidad para inhibir una respuesta verbal automática. Por tanto, se trata de medidas que no engloban todo el concepto de funciones ejecutivas. Quizás otros instrumentos, como el test de clasificación de cartas de Wisconsin (Heaton, Chelune, Talley, Kay y Curtis, 1993) o el test de juego (Bechara, Damasio, Damasio y Anderson, 1994) o, incluso, un protocolo completo para su evaluación (Tirapu, Muñoz-Céspedes, Pelegrín y Albéniz, 2005) aportarían una valoración más exhaustiva de estas funciones mentales.

En suma, los resultados obtenidos no permiten confirmar ninguna de las hipótesis sobre el deterioro planteadas en la introducción. Así, no se encuentra apoyo para la hipótesis del hemisferio derecho puesto que las habilidades visoconstructivas están preservadas. Los resultados tampoco son indicativos de un déficit cognoscitivo generalizado, ya que las alteraciones halladas afectan exclusivamente a la memoria. Por último, tampoco se avala la existencia de un deterioro de predominio frontal, dado que el rendimiento en las pruebas aplicadas para evaluar funciones ejecutivas es normal. En cualquier caso, se trata de unos resultados preliminares que deben confirmarse por otros estudios con muestras más amplias.

\section{REFERENCIAS BIBLIOGRÁFICAS}

Acker, W., Ron, M.A., Lishman, W.A. y Shaw, G.K. (1984). A multivariate analysis of psychological, clinical and CT scanning measures in detoxified chronic alcoholics. British Journal of Addiction, 79, 293-301.

Ambrose, M.L., Bowden, S.C. y Whelan, G. (2001). Working memory impairments in alcohol-dependent participants without clinical amnesia. Alcoholism: Clinical and Experimental Research, 25, 185-191.

American Psychiatric Association, (2000). Diagnostic and statistical manual of mental disorders ( $4^{\text {th }}$ ed.rev.). Washington, D.C. APA.

Baddeley, A.D. (1993). Working memory or working attention? En A.D. Baddeley, L. Weiskrantz (Eds.). Attention: Selection, Awareness, and Control. A tribute to Donald Broadbent. Oxford. Clarendon Press. 
Beatty, W.W., Hames, K.A., Blanco, C.R., Nixon, S.J. y Tivis, L.T. (1996). Visuospatial perception, construction and memory in alcoholism. Journal of Studies on Alcohol, 57, 136-143.

Beatty, W.W., Katzung, V.,M., Moreland, V.J. y Nixon, S.J. (1995). Neuropsychological performance of recently abstinen alcoholics and cocaine abusers. Drug and Alcohol Dependence, 37, 247-253.

Beatty, W.W., Tivis, R., Scott, H.D., Nixon, S.J. y Parsons, O.A. (2000). Neuropsychological deficit in sober alcoholics: influences of chronicity and recent alcohol consumption. Alcoholism: Clinical and Experimental Research, 24, 149-154.

Bechara, A., Damasio, H., Damasio, A.R. y Anderson, S.W. (1994). Insensitivity to future consequences following damage to human prefrontal cortex. Brain, 50, 7-15.

Becker, J.T. y Jaffe, J.H. (1984). Impaired memory for treatment relevant information in inpatient men alcoholics. Journal of Studies on Alcohol, 45, 339-343.

Bertera, J.H. y Parsons, O.A. (1978). Impaired visual search in alcoholics. Alcoholism: Clinical and Experimental Research, 2, 9-14.

Brandt, J., Butters, N., Ryan, C. y Bayog, R. (1983). Cognitive loss and recovery in long-term alcohol abusers. Archives of General Psychiatry, 40, 435-442.

Calvo, H. (2003). Alcohol y neuropsicología. Trastornos Adictivos, 5, 256-268.

Cermak, L.S. y Peck, E. (1982). Continuum versus premature aging theories of chronic alcoholism. Alcoholism: Clinical and Experimental Research, 6, 89-95.

Chan, A.W. (1994). Detection by the CAGE of alcoholism or heavy drinking in primary care outpatients and the general population. Journal of Substance Abuse, 6, 123-135.

Ciesielki, K.T., Waldorf, A.V. y Jung, R.E. (1995). Anterior brain deficits in chronic alcoholism. Cause or effect? Journal of Nervous and Mental Disease, 183, 756-761.

Corral, M.M., Rodríguez, S. y Cadaveira, F. (2002). Perfil neuropsicológico de alcohólicos con alta densidad familiar de alcoholismo tras abstinencia prolongada: hallazgos preliminares. Revista Española de Drogodependencias, $27,148-158$.

Dawson, L.K. y Grant, I. (2000). Alcoholics' initial organizational and problem solving skill predict learning and memory performance on the ReyOsterrieth Complex Figure. Journal of the International Neuropsychological Society, 6, 12-19.

Donovan, D.M., Kivlahan, D.R. y Walker, R.D. (1984). Clinical limitations of neuropsyhological testing in predicting treatment outcome among alcoholics. Alcoholism: Clinical and Experimental Research, 8, 470-475.

Eckardt, M.J., File, S.E., Gessa, G.L., Grant, K.A., Guerra, C., Hoffman, P.L., Kalant, H., Koob, G.F., Li, T.K. y Tabakoff, B. (1998). Effects of moderate alcohol consumption on the central nervous system. Alcohol Clinical and Experimental Research, 22, 998-1040.

Eckardt, M.J., Stapleton, J.M., Rawlings, R.R., Davis, E.Z. y Grodin, D.M. (1995). Neuropsychological functioning in detoxified alcoholics between 18 and 35 years of age. American Journal of Psychiatry, 152, 53-59.

Ellenberg, L., Rosenbaum, G., Goldman, M.S. y Whitman, R.D. (1980). Recover ability of psychological functions following alcohol abuse: lateralization effects. Journal of Consulting and Clinical Psychology, 48, 503-510.

Fein, G., Bachman, L., Fisher, S. y Davenport, L. (1990). Cognitive impairments in abstinent alcoholics. Addiction Medicine, 15, 531-537.

Feuerlein, W., Ringer, C.H. y Kufner, K.A. (1977). Diagnose des alkoholismus: der müncher alkoholismus test (MALT). München Med Woschenchr, 119, 1275-1282.

Giancola, P.R. y Moss, H.B. (1998). Executive cognitive functioning in alcohol use disorders. Recent developments in alcoholism, vol.14, The consequences of alcoholism, New York. Plenun Press.

Goldman, M.S., Williams, D.L. y Klisz, D.K. (1983). Recovery of psychological functioning following alcohol abuse: prolonged visual-spatial dysfunction in older alcoholics. Journal of Consulting and Clinical Psychology, 51, 370378.

Heaton, R.K., Chelune, C.J., Talley, J.L., Kay, G.G. y Curtis, G. (1993). Wisconsin Card Shorting Test. Odessa FL: Psychological Assessment Resources. (Edición Española. TEA, 1997)

Hesselbrock, M.N., Weidenman, M.A. y Reed, H.B. (1985). Effects of age, sex, drinking history and antisocial personality on neuropsychology of alcoholics. Journal of Studies on Alcohol, 46, 313-320.

Hightower, M.G. y Anderson, R.P. (1986). Memory evaluation of alcoholics with Russell's revised wechsler memory Scale. Journal of Clinical Psychology, 42, 1000-1005.

Horner, M.D., Waid, L.R., Johnson, D.E., Latham, P.K. y Anton, R.F. (1999). The relationship of cognitive functioning to amount of recent and lifetime alcohol consumption in outpatient alcoholics. Addictive Behaviors, 24, 449-453.

Ihara, H., Berrios, G.E. y London, M. (2000). Group and case study of the dysexecutive syndrome in alcoholism without amnesia. Journal of Neurology, Neurosurgery and Psychiatry, 68, 731-737.

Iruarrizaga, I., Miguel-Tobal, J.J., Cano, A. (2001). Alteraciones neuropsicológicas del alcoholismo crónico. Un apoyo a la hipótesis del continuo. Psicothema, 13, 571-580.

Jarvis, P. y Barth, J. The Halstead-Reitan Neuropsychological Battery. A guide to interpretation and Clinical Applications. Florida, Psychological Assessment Resources, Inc.1994.

Jenkins, R.L. y Parsons, O.A. (1981). Neuropsychological effect of chronic alcoholism on tactual-spatial 
performance and memory in males. Alcoholism: Clinical and Experimental Research, 5, 26-33.

Jones, B. y Parsons, O.A. (1971). Impaired abstracting ability in chronic alcoholics. Archives of General Psychiatry, 24, 71-75.

Krabbendam, L., Visser, P.J., Derix, M.M., Verhey, F., Hofman, P., Verhoe, W., Tuinier, S. y Jolles, J. (2000). Normal cognitive performance in patients with chronic alcoholism in contrast to patients with korsakoff's syndrome. Journal of Neuropsychiatry and Clinical Neuroscience, 12, 44-50.

Landa, N., Fernández-Montalvo, J. y Tirapu, J. (2004). Alteraciones neuropsicológicas en el alcoholismo: una revisión sobre la afectación de la memoria y las funciones ejecutivas. Adicciones, 16, 41-52.

Leber, W.R., Jenkins, R.L. y Parsons, O.A. (1981). Recovery of visual spatial learning and memory in chronic alcoholics. Journal of Clinical Psychology, 37, 192-197.

Mann, K., Günter, A., Setter, F. y Ackerman, K. (1999). Rapid recovery from cognitive deficits in abstinent alcoholics: a controlled test-retest study. Alcohol and alcoholism, 34, 567-574.

McCrady, B.S. y Smith, D.E. (1986). Implications of cognitive impairment for the treatment of alcoholism. Alcoholism Clinical and Experimental Research, 10, 145-149.

Miglioli, M., Butchell, H.A., Campanini, T.y De Risio, C. (1979). Cerebral hemispheric lateralization of cognitive deficits due to alcoholism. Journal of Nervous and Mental Disease, 167, 212-217.

Moshely, H.F., Georgiou, G. y Kahn, A. (2001). Frontal lobe changes in alcoholism: a review of the literature. Alcohol and Alcoholism, 36, 357-368.

Nixon, S.J. y Bowlby, D. (1996). Evidence of alcoholrelated efficiency deficits in an episodic learning task. Alcoholism Clinical and Experimental Research, 20, 2124.

Nixon, S.J., Kujawski, A., Parsons, O.A. y Yohman, J.R. (1987). Semantic (verbal) and figural memory impairment in alcoholics. Journal of Clinical and Experimental Neuropsychology, 9, 311-322.

Nöel, X., Van der Linden, M., Schmidt, N., Sferraza, R, Hanak, C., Le Bon, O., De Mol, J., Kornreich, Ch., Pelc, I. y Verbank, P. (2001). Supervisory Attentional System in nonamnesic alcoholic men. Archives of General Psychiatry, 58, 1152-1158.

Parsons, O.A. (1987). Neuropsychological consequences of alcohol abuse: many questions, some answers. En O. Parsons, N. Butters, P. Nathan, (Eds.). Neuropsychology of Alcoholism: Implications for Diagnoses and Treatment (pp. 153-173). New York. Guildford Press.

Parsons, O.A. y Leber, W.R. (1981). The relationship between cognitive dysfunction and brain damage in alcoholics: Casual interactive, or epiphenomenal? Alcoholism: Clinical and Experimental Research, 5, 326-343.
Peña-Casanova, J. (1991). Programa Integrado de Exploración Neuropsicológica "Test Barcelona." Normalidad, semiología y patología neuropsicológicas. Barcelona. Masson.

Query, W.T. y Berger, R.A. (1980). AVLT memory scores as a function of age among general medical, neurologic and alcoholic patients. Journal of Clinical Psychology, 36, 1009-1012.

Ratti, M.T., Bo, P., Giardini, A. y Soragna, D., (2002). Chronic alcoholism and the frontal lobe: which executive functions are impaired? Acta Neurologica Scandinavica, 105, 276.

Ratti, M.T., Soragna, D., Sibilla, L., Giardini, A., Albergati, A., Savoldi, F. y Bo, P. (1999). Cognitive impairment and cerebral atrophy in "heavy drinkers". Pro Neuropsychopharmacol biol Psychiatry, 23, 243-258.

Rey, A. (1959). Test de copie et de Reproduction de Memore de Figures Géometriques Complexes. París. Les editions Du Centre de Psychologie Appliquée. Adaptación española ( $5^{a}$ ed.), 1987. Madrid. TEA ediciones.

Riege, W.H., Tomaszewewski, R., Lanto, A. y Metter, E.J. (1984). Age and alcoholism: Independent memory decrements. Alcoholism: Clinical and Experimental Research, 8, 42-47.

Rodríguez-Martos, A., Suárez, R. (1984). MALT (Munchner Alkoholismus Test). Validación de la versión española de este test para el diagnóstico de alcoholismo. Revista de Psiquiatría y Psicología Médica, 16, 421-432.

Ryan, C. y Butters, N. (1980). Further evidence for a continuum of impairment encompassing male alcoholic korsakoff patients and chronic alcoholic men. Alcoholism: Clinical and Experimental Research, 4, 190-198.

Stroop, J.R. (1935). Studies of interference in serial verbal reactions. Journal of Experimental Psychology, 18, 643662.

Strub, R.L. y Black, F.W. (1995). The mental status examination in neurology. Philadelphia. Davis.

Sullivan, E.V., Fama, R., Rosenbloom, M.J. y Pfefferbaum, A. (2002). A profile of neuropsychological deficits in alcoholic women. Neuropsychology, 16, 74-83.

Sullivan, E.V., Mathalon, D.H., Zipursky, R.B., KersteenTucker, Z., Knight, R.T. y Pfefferbaum, A. (1993). Factors of the Wisconsin Card Sorting Test as measures of frontal lobe function in schizophrenia and in chronic alcoholism. Psychiatry Research, 46, 175-199.

Sullivan, E.V., Rosenbloom, M.J. y Pfefferbaum, A. (2000). Pattern of motor and cognitive deficits in detoxified alcoholic men. Alcoholism: Clinical and Experimental Research, 24, 611-621.

Tarquini, D. y Masullo, C. (1981). Cognitive impairments and chronic alcohol abuse: A neuropsychological study. Drug and Alcohol Dependence, 8, 103-109. 
Tarter, R.E. (1975). Brain damage associated with chronic alcoholics, Disease of Nervous System, 36, 185-187.

Tarter, R.E. (1976). Neuropsychological investigations of alcoholism. En G. Goldstein y C. Neuringer (Eds.). Empirical studies of alcoholism (pp. 231-256). Cambridge, Mass.Ballinger.

Tarter, R.E. y Alterman, A.I. (1984). Neuropsychological deficits in alcoholics: etiological considerations. Journal of Studies on Alcohol, 45, 1-9.

Thurstone, L. (1938). Primary mental abilities. Chicago. University of Chicago Press

Tivis, L.J. y Parsons, O.A. (1995). An investigation of verbal spatial functioning in chronic alcoholics. Assessment, 2, 285-292.
Tirapu, J., Landa, N. y Lorea, I. (2004). Cerebro y adicción. Pamplona. Gobierno de Navarra.

Tirapu, J., Muñoz Céspedes, J.M., Pelegrín, C. y Albéniz, A. (2005). Propuesta de un protocolo para la evaluación de las funciones ejecutivas. Revista de Neurología, 41, 177-186.

Tsagareli, M.G. (1995). The interhemispheric functional organization on human visuo-spatial perception. Neuroreport, 6, 925-928.

Wechsler, D. (1987). Wechsler Memory Scale-Revised. Manual. San Antonio. Psychological Corporation Harcourt Brace, Inc.

Yohman, J.R., Parsons, O.A., (1985). Intact verbal pairedassociate learning in alcoholics. Journal of Clinical Psychology, 41, 844-851. 
